\title{
BMJ Open Improving reproductive function in women with polycystic ovary syndrome with high-intensity interval training (IMPROV-IT): study protocol for a two- centre, three-armed randomised controlled trial
}

Ida Almenning Kiel (D , ,,2 Sofie Lionett, ${ }^{1,2,3}$ Evelyn Bridget Parr, ${ }^{3}$ Helen Jones, ${ }^{4}$ Maria Aurora Hernandez Røset, ${ }^{2,5}$ Øyvind Salvesen, ${ }^{6}$ Eszter Vanky, ${ }^{2,5}$ Trine Moholdt ${ }^{1,2}$

To cite: Kiel IA, Lionett S, Parr EB, et al. Improving reproductive function in women with polycystic ovary syndrome with high-intensity interval training (IMPROV-IT): study protocol for a two-centre, three-armed randomised controlled trial. BMJ Open 2020;10:e034733. doi:10.1136/ bmjopen-2019-034733

- Prepublication history for this paper is available online. To view these files, please visit the journal online (http://dx.doi. org/10.1136/bmjopen-2019034733).

Received 03 October 2019 Revised 15 January 2020 Accepted 04 February 2020
Check for updates

(C) Author(s) (or their employer(s)) 2020. Re-use permitted under CC BY-NC. No commercial re-use. See rights and permissions. Published by BMJ.

For numbered affiliations see end of article.

Correspondence to

Dr Trine Moholdt;

trine.moholdt@ntnu.no

\section{ABSTRACT}

Introduction Polycystic ovary syndrome (PCOS) is a common endocrine disorder in women of reproductive age and the leading cause of anovulatory infertility. Women with PCOS have a 15-fold higher prevalence of infertility, compared with women without PCOS, independent of body mass index (BMI). A healthy lifestyle is recommended to improve overall health and fertility in PCOS but there is limited evidence on the isolated effects of exercise, especially for reproductive outcomes. Previous findings indicate superior metabolic health benefits after vigorous compared with moderateintensity exercise. Our primary aim is to determine the effect of high-intensity interval training (HIT) on menstrual frequency, as a proxy of reproductive function, in women with PCOS.

Methods and analysis The study is a two-centre, randomised, controlled trial with three parallel groups. Women ( $n=64)$ from Trondheim (Norway) and Melbourne (Australia) with PCOS according to the Rotterdam criteria will be randomly allocated (1:1:1) to high-volume HIT, low-volume HIT or a control group with no exercise after stratifying for $\mathrm{BMI}<$ or $\geq 27 \mathrm{~kg} /$ $\mathrm{m}^{2}$ and study centre. Measurements for study end points will be undertaken at baseline, after a 16 week exercise intervention and at 12 months following baseline assessments. The primary outcome measure is menstruation frequency, measured as the number of self-reported menstrual bleedings divided by the number of expected menstrual bleedings during a 12-month period. Secondary outcome measurements include markers of cardiovascular, metabolic and reproductive health, as well as quality of life and adherence to and enjoyment of exercise.

Ethics and dissemination The Regional Committee Medical Research Ethics, Norway, and The Australian Catholic University Human Research Ethics Committee, Australia, have approved the trial protocol. This trial will provide new insight regarding the impact of exercise on fertility in PCOS. We expect this trial to contribute to new
Strengths and limitations of this study

- This will be the first randomised controlled trial to determine the isolated effects of two different highintensity interval training protocols on reproductive and health-related outcomes in women with polycystic ovary syndrome, with a follow-up time of 12 months.

- The exercise intervention is controlled and monitored through partly supervised sessions for 16 weeks.

- We will include women within all body mass index categories.

- Investigators will not be blinded for all assessments due to the difficulty blinding investigators and participants to a behavioural intervention.

- Menstrual frequency is only a proxy for reproductive function, and we will not undertake ovulation monitoring.

therapeutic exercise strategies as part of clinical care for women with PCOS.

Trial registration number Clinical trial gov NCT02419482.

\section{INTRODUCTION}

Polycystic ovary syndrome (PCOS) is a complex endocrine disorder ${ }^{12}$ affecting $8 \%-13 \%$ of reproductive-aged women. ${ }^{3}$ The ovulatory disturbance is a central diagnostic feature of PCOS,${ }^{4}$ and the syndrome is recognised as the leading cause of anovulatory infertility and menstrual disorders. ${ }^{5}$ A large community-based cohort study reported a $72 \%$ prevalence of infertility among women with PCOS, compared with $16 \%$ among women without PCOS. ${ }^{6}$ 
According to the most recent guidelines for the assessment and management of PCOS, lifestyle intervention is regarded as first-line therapy to manage reproductive and metabolic outcomes. ${ }^{4}$ In addition to reproductive dysfunctions, PCOS is associated with a number of adverse cardiovascular, metabolic and psychological outcomes across all categories of body mass index (BMI). ${ }^{2}{ }^{7-9}$ Obesity seems to exacerbate the clinical features in PCOS, but also weight-independent insulin resistance is strongly implicated in the aetiology of PCOS, contributing to the reproductive and metabolic complications. ${ }^{10-13}$ Approximately $40 \%$ of women with PCOS have a normal BMI $\left(<25 \mathrm{~kg} / \mathrm{m}^{2}\right)$ and the prevalence of insulin resistance among lean women with PCOS is estimated to be around 75\%. ${ }^{14}$ Adipose tissue dysfunction, such as hypertrophic adipocytes and impairments in lipolysis and insulin action, plays a central role in the metabolic abnormalities observed in PCOS. ${ }^{15}$ Hypertrophic adipocytes are more susceptible to inflammation and chronic low-grade inflammation in PCOS. ${ }^{16}$ There is limited research on the effect of exercise training on adipose tissue function and lowgrade inflammation in PCOS.

Exercise training positively affects metabolic, cardiovascular and psychological outcomes in women with PCOS, but the effect on fertility is unclear. ${ }^{41217-19}$ Some trials $^{20-24}$ and systematic reviews ${ }^{12} 25$ report improved ovulation or menstruation frequency after a period of exercise training or after a combined exercise-diet intervention. ${ }^{26}$ Two of these studies indicate a weightindependent effect of exercise on fertility, suggesting insulin sensitivity to be a key factor. ${ }^{21}{ }^{23}$ Only one study to date has investigated the effect of exercise on menstruation frequency in women with PCOS having normal or low BMI $\left(<25 \mathrm{~kg} / \mathrm{m}^{2}\right){ }^{22}$ In that study, they found a significant improvement in menstruation frequency (from 48 to 27 days) after 8 weeks (three sessions per week) of aerobic and resistance training. However, 8 weeks follow-up may be too short to determine any effect of exercise training on menstrual frequency. Furthermore, inconsistent reporting of fertility-related outcomes, small sample sizes and short intervention periods, make it difficult to interpret the effect of exercise on fertility. ${ }^{241225}$

Despite growing evidence on health benefits of exercise training in PCOS, ${ }^{42} 27$ there is a lack of welldesigned randomised controlled trials of different exercise protocols including long intervention and follow-up periods to determine the isolated effect of exercise on fertility outcomes

. Most studies to date have only included overweight/ obese participants and less is known about the potential benefits in women with PCOS who have BMI $\leq 25 \mathrm{~kg} / \mathrm{m}^{2}$. Although moderate-intensity exercise provides health benefits, only vigorous, and not moderate-intensity, physical activity was associated with reduced odds of insulin resistance and metabolic syndrome in a crosssectional study of women with PCOS. ${ }^{18}$ Indeed, there is an increased focus on high-intensity interval training (HIT) as a means of improving insulin sensitivity ${ }^{28}$ and cardiorespiratory fitness ${ }^{29-31}$ in clinical populations, including PCOS. ${ }^{32}$ HIT involves brief, repeated work bouts of relatively intense exercise separated by periods of rest or low-intensity exercise. Several different HIT protocols exist, and they can broadly be divided into 'high-volume' (HV) and 'low-volume' (LV) HIT. One of the most common HV-HIT protocols is the Norwegian $4 \times 4$ min HIT protocol, which induces superior improvements in cardiorespiratory fitness compared to work-matched moderate-intensity training in both healthy individuals ${ }^{33}$ and in various patient groups. ${ }^{29} 34$ LV-HIT typically consists of $\leq 10 \mathrm{~min}$ of intense exercise within an exercise session lasting $\leq 30 \mathrm{~min}$ in total, such that the total weekly training time commitment is markedly lower than the current public health guidelines. ${ }^{35}$ LV-HIT could therefore have the potential to overcome the most common barriers for women in fertile age, such as time commitment. ${ }^{36}$ LV-HIT can improve glycaemic control in people with type 2 diabetes. ${ }^{37}$ We have previously reported improved insulin sensitivity (measured with homeostatic assessment of insulin resistance, HOMA-IR), endothelial function and body composition after 10 weeks of HIT in women with PCOS, without any changes in body mass. ${ }^{32}$ However, in that pilot study, the participants undertook both LV-HIT and HV-HIT and we did not determine the effects of exercise training on any reproductive outcomes. Based on the positive results from our pilot study with a combined HV-HIT and LV-HIT protocol, we will now compare the two different HIT protocols to investigate potential differences in health outcomes.

Here we describe the design, methodology and potential clinical significance of the 'IMproving Reproductive function in women with Polycystic OVary syndrome by high-intensity Interval Training (IMPROV-IT) trial'.

\section{AIMS}

The primary aim of the IMPROV-IT trial is to test the hypothesis that 16 weeks of semi-supervised HIT, followed by home-based HIT for 36 weeks, will increase menstruation frequency, as a proxy measure of ovulation, during 1 year of follow-up in women with PCOS, compared with a non-exercising control group.

Secondary aims are to determine if there are improvements in: ovarian morphology, insulin sensitivity, endothelial function, intima-media thickness, body composition, cardiorespiratory fitness, oxidative capacity, circulation markers of reproductive and metabolic health, low-grade systematic inflammation (in blood and adipose tissue), adipose tissue morphology and function, and quality of life, after 16 weeks of semisupervised HIT, as well as after the following 36 weeks of home-based HIT.

Additionally, we will record the adherence to exercise training and enjoyment of two different HIT protocols 


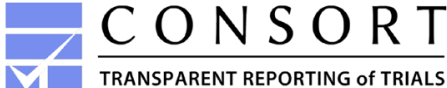

CONSORT 2010 Flow Diagram

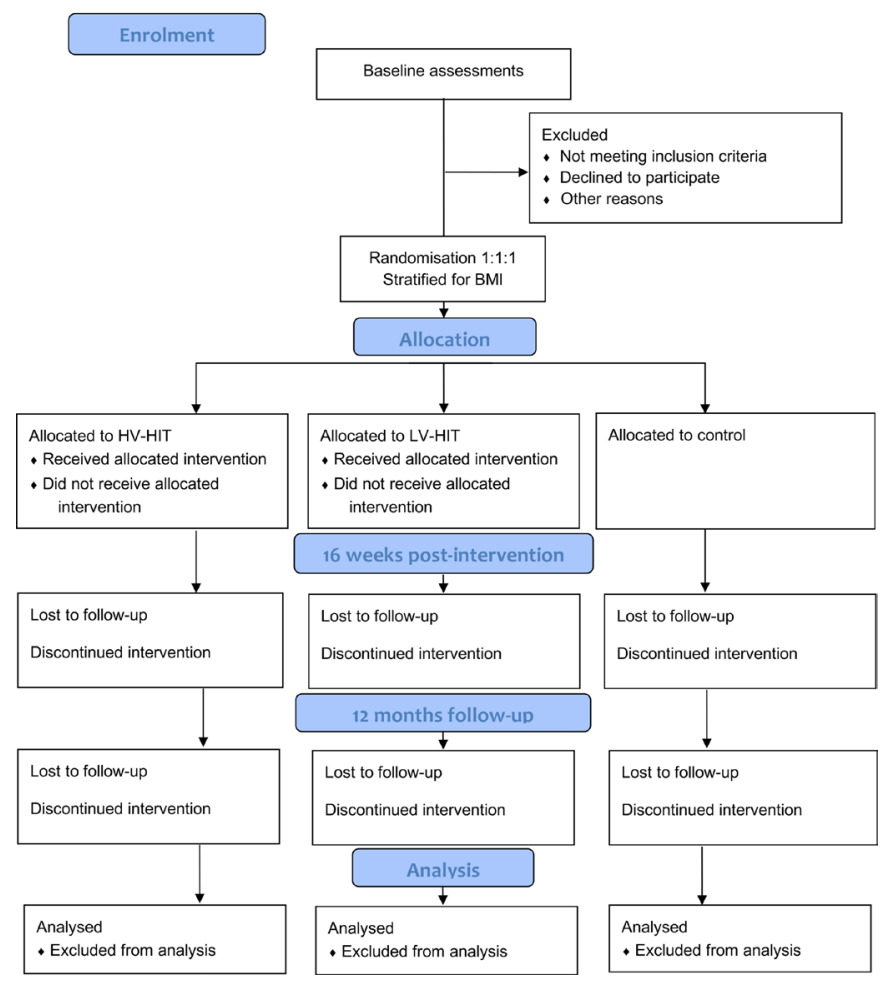

Figure 1 Consort flow diagram. BMI, body mass index; HVHIT, high-volume high-intensity interval training; LV-HIT, lowvolume high-intensity interval training.

and assess if the two protocols induce different effects on the aforementioned measures.

\section{METHODS AND ANALYSIS}

\section{Study setting and recruitment}

This is a two-centre, randomised controlled trial with three parallel groups; two training intervention groups and one control group. The two study centres are the Norwegian University of Science and Technology (NTNU) in Trondheim, Norway and the Australian Catholic University (ACU) in Melbourne, Australia. Testing and training will take place in the university research laboratories in both Norway and Australia. Participants will be recruited from the public announcement at hospitals and university homepages, at local stores, public places and social media. All participants will sign a written, informed consent. All participants will have a project-specific code that will be used during all analyses of the data. The procedures for data entry, coding and storage have been approved by the Regional Committee Medical Research Ethics in Mid-Norway.

\section{Participants}

To be eligible for inclusion in the study, women will have to meet the following criteria:
- Aged 18-45 years old.

- PCOS diagnosis according to the Rotterdam criteria, ${ }^{38}$ as confirmed by a gynaecologist, endocrinologist or general practitioner. Thus, a minimum of two of the following criteria have to be present: (1) polycystic ovary morphology (12 or more $2-9 \mathrm{~mm}$ follicles or $>10 \mathrm{~mL}$ in volume, in at least one ovary), (2) hyperandrogenism (either clinical signs as hirsutism or acne, or biochemical), and (3) oligo/amenorrhoea. Oligomenorrhoea is defined as an intermenstrual interval $>35$ days and/or $\leq 9$ menstrual bleeding in the past year. Amenorrhoea is defined as no vaginal bleeding the last 3 months for women with a previously regular menstrual cycle; no vaginal bleeding the last 12 months for women with an irregular menstrual cycle. $^{39}$ 40 Hirsutism will be defined as modified Ferriman Gallwey score ${ }^{41} \geq 8{ }^{4}$

- At the Norwegian centre, all participants will be screened at baseline for polycystic ovaries, hyperandrogenism and menstruation frequency to confirm the PCOS diagnosis. At the Australian centre, participants will show an ultrasound scan no older than 8 years performed by their general practitioner, gynaecologist or endocrinologist confirming polycystic ovaries. Ferriman Gallway score for hirsutism/hyperandrogenism and information about their menstrual cycle will be obtained before entering the study to confirm PCOS diagnosis.

- Undertaking less than 2 weekly endurance exercise training sessions with an intensity that induce heavy breathing.

Women will be excluded from the study if they meet any of the following criteria:

- Current treatments with hormonal contraceptives including Mirena intrauterine device.

- Insulin sensitisers or drugs known to affect gonadotropin or ovulation (with a washout period of 3 months prior to inclusion).

- On-going pregnancy or breastfeeding within 24 weeks.

- Cardiovascular disease or other endocrine disorders (eg, congenital adrenal hyperplasia, Cushing syndrome or androgen-secreting tumours).

\section{Randomisation and allocation}

Participants will be allocated 1:1:1 to HV-HIT, LV-HIT, or control after stratifying for BMI $<$ or $\geq 27 \mathrm{~kg} / \mathrm{m}^{2}$ and study centre (figure 1). A computer random number generator developed and administered at the Faculty of Medicine, Department of Public Health and General Practice, NTNU, Trondheim, Norway, will be used at both study centres. The investigators will be informed about the allocation results by e-mail after the registration of new participants.

\section{Interventions}

The first 16 weeks of the exercise training will be semisupervised, with the remaining 36 weeks of the intervention organised as home-based exercise training without 
any supervision. Supervised exercise interventions are effective, but do not resemble a real-life setting, and the long-term adherence often falls once the supervised exercise programme ends. Unsupervised exercise programme are more easily implemented and flexible, but adherence is often low. During the first 16 weeks, the participants will perform at least 1 weekly supervised exercise session at the study centres. They will be given the opportunity to attend up to 3 weekly supervised exercise sessions but can also choose to do one or two of the weekly sessions as home-based training. The participants will be encouraged to continue to complete at least 2 weekly sessions (homebased and unsupervised) for the remaining 36 weeks of the study period. Participants receive a heart rate monitor that they will wear on all sessions and will be required to register all their exercise sessions during the whole study period through an online exercise-training diary (www.polar.flow.com). The researchers will have access to all sessions via the same online tool and will supervise exercise adherence at the home-based sessions; however, no motivational support or instructions will be provided during the follow-up period. We will be advising women in the control group to continue their habitual physical activity and inform them about the current recommendations of a minimum of $150 \mathrm{~min}$ of weekly moderateintensity physical activity.

\section{HIT protocols}

The LV-HIT protocol consists of a 10 min warm-up at light to moderate intensity at $60 \%-70 \%$ of $\mathrm{HR}_{\max }$ followed by ten 1 min work bouts at the maximal intensity the participants can complete for $1 \mathrm{~min}$. In the initial training session, the intensity will be set corresponding to $100 \%$ of the workload the participant reached at the baseline $\mathrm{VO}_{2}$ max test. Participants will be instructed to try reaching $90 \%$ of maximal heart rate $\left(\mathrm{HR}_{\max }\right)$ during the third or fourth work bout, based on previous findings from Little et $a l .{ }^{37}$ The work bouts are separated by 1 min of passive or low-intensity recovery, trying to reach $60 \%-70 \%$ of $\mathrm{HR}_{\max }$ The training session is terminated after a 3 min cool-down at $60 \%-70 \%$ of $\mathrm{HR}_{\max }$. The total exercise time is $32 \mathrm{~min}$ (figure 2A).
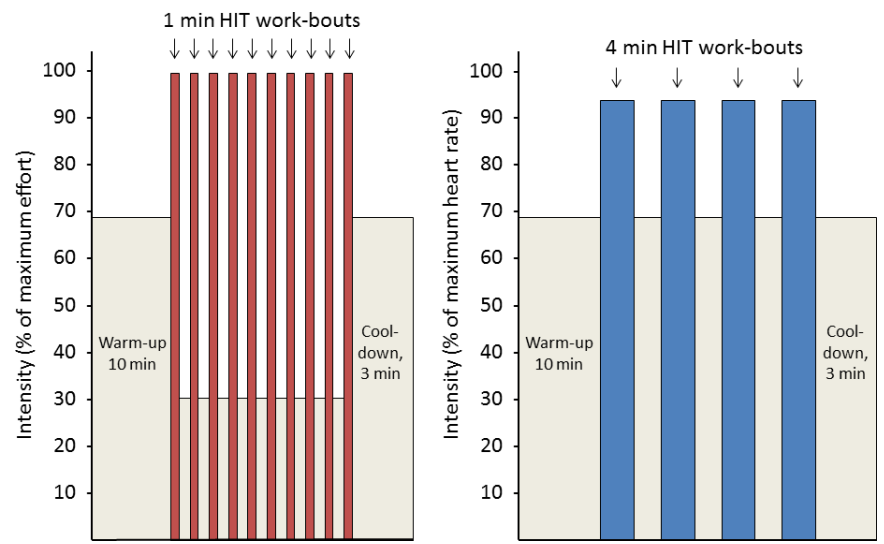

Figure 2 HIT protocol. Panel A: low-volume hit; Panel B: high-volume hit. HIT, high-intensity interval training.
The HV-HIT protocol consists of a $10 \mathrm{~min}$ warm-up at $60 \%-70 \%$ of $\mathrm{HR}_{\max }$, followed by four $4 \mathrm{~min}$ work bouts reaching $90 \%-95 \%$ of $\mathrm{HR}_{\max }$, separated by a 3 min active recovery of running/walking at $60 \%-70 \%$ of $\mathrm{HR}_{\max }$. The training session is terminated after a 3 min cool-down at $60 \%-70 \%$ of $\mathrm{HR}_{\max }$. The total exercise time is $38 \mathrm{~min}$ (figure 2B).

The LV-HIT and HV-HIT are not matched for time, mean workload and energy expenditure. HIT will be performed as treadmill walking or running. Heart rate monitors (Polar M400) will be used on all sessions, including on the home-based sessions, and the exercise intensity will be estimated based on their $\mathrm{HR}_{\max }$ during the $\mathrm{VO}_{2} \max$ test at baseline. ${ }^{42}$ Where required (due to injury, pain or discomfort), the participants will be able to exercise on other ergometers (bikes or elliptical machines). Participants can also choose to perform some of the sessions as outdoor running/uphill walking. We will adjust the absolute workload of both LV-HIT and HV-HIT throughout the intervention period to account for improvements in fitness.

\section{Outcome measures}

Outcomes will be assessed at baseline, at 16 weeks (post intervention) and at 12 months from baseline (follow-up) in Trondheim, Norway. In Melbourne, Australia, measurements will be assessed at baseline and after 16 weeks, and only questionnaires and menstrual frequency will be assessed until 12 months from baseline. Participants will be asked not to exercise for $>48$ hours prior to the test visits in the laboratory and to abstain from caffeine intake for 24 hours prior to the tests. Measurements will be undertaken during the follicular phase of the menstrual cycle (1-7 days after first bleeding) in women with a regular menstrual cycle.

\section{Menstrual frequency}

The primary outcome measure is menstrual frequency. Participants will register the first day of their menstrual cycle and length (number of days) during the 12-month study period. They will send their menstruation dairy to the study personnel after each menstrual cycle, and the study personnel will send out reminders to fill out the diary. In addition, participants will complete a questionnaire about their menstrual cycle at each assessment point (baseline, 16 weeks and 12 months). We will compare the number of menstrual bleedings between the groups, measured as the number of observed menstrual bleedings divided by the number of expected menstrual bleedings ( $\mathrm{n}=13$, assuming a cycle interval of 28 days) during a 12-month period. The menstrual frequency is to be measured as a proxy for ovulation. Menstrual frequency assessments will be undertaken non-blinded for the study personnel.

\section{Ovarian morphology}

Ovarian morphology will only be assessed at the site in Norway. The ultrasound assessments of the ovaries include 
ovarian volume in $\mathrm{mL}$ (with and without a dominating follicle), dominating follicle volume in $\mathrm{mL}$, number of follicles in each ovary, diameter and distribution of follicles. We will use a multifrequency transvaginal transducer on all measurements. An experienced gynaecologist (MAHR) will perform all imaging and measurements. Ovarian morphology assessments will be undertaken blinded for group allocation.

\section{Fertility and pregnancies}

Background information regarding fertility will be registered by questionnaires. These include questions about number of children, natural or assisted fertilisation, if the participants are actively seeking pregnancy or have tried to become pregnant, for how long they have tried to become pregnant, and if they have experienced miscarriages or had abortions. We will report the number of pregnancies during the intervention period, including during the 12 months of follow-up. Questionnaires will be undertaken blinded, whereas the number of pregnancies will be undertaken non-blinded for the study personnel.

\section{Blood biochemistry and insulin sensitivity}

Blood samples will be obtained after an overnight fast (no food or fluid intake except water 12 hours prior to assessments) at each time point. Analysis of fasting venous blood samples will include measurements of $17-\mathrm{OH}$ progesterone (baseline only), prolactin (baseline only), haemoglobin, glycated haemoglobin, blood lipids (total cholesterol, high-density lipoprotein cholesterol, lowdensity lipoprotein cholesterol and triglycerides), blood glucose, white blood cell count (neutrophils, basophils, eosinophils, lymphocytes and monocytes) and leucocytes. Additional blood (including buffy coat) will be stored for later analyses. These later analyses will likely include, but are not limited to, androstenedione, anti-Müllerian hormone, sex hormone-binding globulin, testosterone, insulin, leptin, adiponectin and micro-RNAs. We will also obtain two Tempus RNA tubes from each participant for later assessments of gene expression.

After fasting blood samples, glycaemic control will be measured using a 2-hour oral glucose tolerance test (OGTT). The participants will consume $75 \mathrm{~g}$ of glucose diluted in $250 \mathrm{~mL}$ water. Blood will be sampled for insulin and glucose measurements at 0 (prior to the OGGT), 30, 60,90 and $120 \mathrm{~min}$ from an indwelling catheter. Glycaemic control will be calculated as total area under the curve (AUC) and incremental area under the curve (iAUC; using fasting concentrations as baseline values) using the trapezoid method, for glucose and insulin concentrations, and peak concentrations during the OGTT. Insulin sensitivity will be estimated using the HOMA-IR; fasting serum insulin in $\mu \mathrm{U} / \mathrm{mL} \times$ fasting plasma glucose in $\mathrm{mmol} / \mathrm{L} / 22.5{ }^{43}$

Biological materials collected at NTNU will be stored in the Regional Research Biobank at St Olav's Hospital (Biobank1, https://biobank1.no/nb/). Biological materials from collected at ACU will be stored locally until data collection is completed in Australia, and later sent to Norway. Sample collection and handling are performed in accordance with hospital/laboratory standard procedures. Blood sampling and OGTT will be undertaken blinded in Norway and unblinded in Australia.

\section{Cardiorespiratory fitness and substrate utilisation}

Peak oxygen uptake will be measured on a treadmill, using indirect calorimetry (Oxycon Pro, Jaeger, Germany in Norway/TrueOneRMR, Parvo Medics, USA in Australia). Participants will walk or run until voluntary exhaustion using an individualised protocol. After a $10 \mathrm{~min}$ walking warm-up, the test will start by walking at moderate intensity for $3 \mathrm{~min}$. The speed or inclination will then be increased every $1-2 \mathrm{~min}$, by $0.5-1.0 \mathrm{~km} / \mathrm{h}$ or $1 \%-2 \%$. The cardiorespiratory fitness test will be considered successful with a plateau in $\mathrm{VO}_{2}$ with a further increase in workload, and a respiratory exchange ratio $\geq 1.05$. Peak oxygen uptake will be calculated as the highest consecutive $30 \mathrm{~s}$ measured, both absolute $(\mathrm{mL} / \mathrm{min})$ and relative $(\mathrm{mL} / \mathrm{min} / \mathrm{kg})$. The maximal heart rate obtained during the exercise test at baseline will serve as the basis for calculating the intensity during the HIT sessions.

After an overnight fast (no food or fluid intake except water 12 hours prior to assessments), participants will complete a submaximal test on a treadmill. The protocol includes a 20 min warm-up, followed by 20 min steadystate expired gas sampling at $60 \%$ of peak oxygen uptake. We will ask participants to record their diet the day before the baseline test of oxidative capacity and to repeat this diet before the subsequent measures (at 16 weeks and 12 months). Fat oxidation rates $(\mathrm{g} / \mathrm{min})$ will be calculated from $5 \mathrm{~min}$ of stable oxygen uptake at the final stage of the test (during the last $10 \mathrm{~min}$ ), using the following equation: $1.695 \times \mathrm{VO}_{2}-1.701 \times \mathrm{VCO}_{2}$, ${ }^{44}$ where $\mathrm{VO}_{2}$ is oxygen uptake and $\mathrm{VCO}_{2}$ is the volume of expired carbon dioxide. Assessments of these outcomes will be undertaken non-blinded for the study personnel.

\section{Adipose tissue composition, morphology and function}

Abdominal and gluteal subcutaneous adipose tissue biopsies $(\sim 300-500 \mathrm{mg})$ will be collected under local anaesthesia ( $1 \%$ xylocaine) using a $14-\mathrm{G}$ sterile needle. For a subsample of participants, $\sim 80 \mathrm{mg}$ of each biopsy will be allocated for immediate analyses of mitochondrial respiration using high-resolution respirometry (Oxygraph-2K, Oroboros, Austria). Approximately $200-300 \mathrm{mg}$ will be snap-frozen in liquid nitrogen and stored at $-80^{\circ} \mathrm{C}$ for later analyses, such as micro RNAs, gene expression and protein profiling. The remaining tissue will be immediately fixed in phosphate-buffered formalin, processed and embedded in paraffin and sectioned at $4 \mu \mathrm{m}$ for morphology and inflammation analyses. The sections will be incubated with $\mathrm{CD} 45$ and $\mathrm{CD} 68$ antibodies to detect leucocytes and macrophages. These sections will be captured with an EVOS FL Auto 2 Imaging System (ThermoFisher Scientific, USA) and analysed with the 
open-source software ImageJ (Fiji). ${ }^{45}$ Assessments of these outcomes will be undertaken blinded.

\section{Physical activity and diet}

We will monitor physical activity by questionnaires and 5-day activity monitoring (Sensewear Armband, APC Cardiovascular, UK) and record diet through a 4-day diet recall, at each measurement point. Assessments of physical activity and diet will be undertaken blinded.

\section{Quality of life}

Quality of life will be assessed using the Polycystic Ovary Syndrome Questionnaire. ${ }^{46}$ Assessment of this outcome will be undertaken blinded.

\section{Anthropometrics and body composition}

Anthropometric measurements will be conducted when participants are fasted (no food or fluid intake except water 12 hours prior to assessments). Height will be measured standing, without shoes using a standard stadiometer. Total body mass and body composition will be measured wearing light clothing with no metal items and without shoes or socks using bioelectrical impedance analysis (InBody720 bioimpedance scale, Biospace CO, Korea) in Norway and dual-energy X-ray absorptiometry (DXA; GE Lunar iDXA Pro, encore software version 16, General Electric, Boston, Massachusetts, USA) in Australia. For the DXA scan, participants are required to lay supine on the scanning bed for the duration of the scan, which is approximately $15 \mathrm{~min}$ with one or two scans depending on the body shape of the participant. Waist and hip circumference will be measured to the nearest $0.5 \mathrm{~cm}$ horizontally at the level of the umbilicus, while standing and at normal expiration, using a metric tape. Assessments of these outcomes will be undertaken nonblinded for the study personnel.

\section{Blood pressure and resting heart rate}

Blood pressure and resting heart rate will be measured in the seated position after $15 \mathrm{~min}$ rest with an automatic blood pressure device three times on the left arm (diastolic and systolic, in $\mathrm{mm} \mathrm{Hg}$ ). The mean of the three measurements will be used to calculate blood pressure and resting heart rate. Assessments of these outcomes will be undertaken blinded in Norway and unblinded in Australia.

\section{Endothelial function and intima-media thickness}

Endothelial function and intima-media thickness (IMT) will be assessed at the Norwegian site only. Following a 20 min supine rest period, the diameter of the brachial artery will be imaged (12 MHz Doppler probe, GE Vingmed Ultrasound AS, Horten, Norway). A cuff will be placed around the forearm (immediately distal to the olecranon) to produce the stimulus of forearm ischaemia. When an optimal image is obtained, the probe will be held stable and the ultrasound parameters will be set to optimise the longitudinal, B-mode image of the lumen-arterial wall interface. The ultrasound will also be used to attain simultaneous continuous Doppler velocity using the lowest possible insonation angle $\left(60^{\circ}\right)$. A recording of resting diameter and velocity will be taken for $1 \mathrm{~min}$, then the forearm cuff will be inflated $(>200 \mathrm{~mm}$ $\mathrm{Hg}$ ) for $5 \mathrm{~min}$. Both diameter and velocity recordings will resume $30 \mathrm{~s}$ before cuff deflation and continue for $3 \mathrm{~min}$ after deflation. ${ }^{47}$ This flow-mediated dilation (FMD) test is a measure of endothelial function. We will also measure carotid artery IMT in three angles: transversal, longitudinal and anterolateral using ultrasound $(12 \mathrm{MHz}$ Doppler probe, GE Vingmed Ultrasound AS, Horten, Norway). Measurements of the diameter will be imaged $1 \mathrm{~cm}$ below the bifurcation on the right side. Images will be obtained where the near wall is clearly visualised, with a double-line pattern and at the minimal diameter during the cardiac cycle as previously described elsewhere. ${ }^{48}$ The same person (IAK) will perform all the FMD and IMT measurements. Assessments of these outcomes will be undertaken non-blinded for the study personnel.

\section{Enjoyment}

A subgroup of participants (the last 40 to be included in the trial) allocated to one of the training groups will complete a Physical Activity Enjoyment Scale (PACES) ${ }^{49}$ as well as The Borg's scale ${ }^{50}$ to assess enjoyment and perceived exertion at one supervised weekly training session. Assessments of these outcomes will be undertaken non-blinded for the study personnel.

\section{Sample size and statistical analysis}

We computed the sample size for a one-way analysis of variance test with three groups. In women with PCOS, a menstrual frequency of on average 4.5 menstrual bleeding during 1 year is expected. ${ }^{51}$ With a statistical power of $80 \%$, a significance level of 0.05 and a SD of two menstrual bleeding during a 12-month period, we calculated that 48 women will be required to detect an increase in menstrual bleeding to 7.5 in the intervention groups. ${ }^{26}$ Because of the non-normality of menstrual frequency, it may be necessary to use a non-parametric test. Non-parametric tests require more participants and we, therefore, added $15 \%$ to the required sample size. Additionally, to allow for expected dropouts of $10 \%-15 \%$, we aim to include 64 women in the study.

We will perform all the statistical analyses blinded for group allocation. The primary analysis will be a comparison between groups for the number of menstrual bleeding during 12 months. We will adjust for the selfreported menstrual frequency at baseline. We will include all women who have reported menstrual frequency in the primary analysis, independent of adherence to the interventions, that is, intention-to-treat analysis. The number of pregnancies during the 16 weeks of intervention and during the 12-month follow-up will be compared between groups. Secondary outcome measures will be compared between groups after 16 weeks and after 12 months and adjusted for baseline values. We will do additional 'per protocol' analyses where we include women in the HIT 
groups that have completed $>75 \%$ of the scheduled exercise sessions during the intervention period (for comparisons after 16 weeks) and a mean minimum of one HIT session/week during the 12-month follow-up (for comparisons at the end of follow-up).

Results will be reported as means with $95 \%$ CIs and/or SD. We will report the effect of HIT as mean changes from baseline to 16 weeks of intervention and after 12 months of follow-up. We will use mixed models to test differences between groups. $\mathrm{P}$ values $<0.05$ will be considered significant for both primary and secondary outcomes. We will also perform subgroup analyses to investigate differences between BMI categories and compare adherence rates and enjoyment of the two HIT protocols.

\section{Blinding}

We are unable to blind group allocation to participants or study personnel due to the nature of the intervention (supervised exercise training). All baseline assessments will be undertaken prior to randomisation and some assessments will be undertaken blinded for group allocation (as outlined for each outcome measure).

\section{Monitoring}

The investigators are responsible for the documentation of any adverse event or serious adverse event. Participants will be told to contact the investigator if they have any unusual symptoms. We will record all medical events during the study in the Case Report Form. In addition, we record serious adverse events in a Serious Adverse Events Report Form. All serious adverse events will be reported to the sponsor within 24 hours after the site has gained knowledge of the event.

\section{Patient and public involvement}

No patients were involved in the development of the research question or design of the study. All participants will be invited to a 1-hour educational session about healthy diet and physical activity. We will also give general information about PCOS in these meetings and encourage the participants to ask questions and give us feedback about relevant topics or issues related to their disorder and to the trial implementation. Individual test results will be disseminated to each participant after testing. We will also send out a summary of the study results to all participants at the completion of the study.

\section{ETHICS AND DISSEMINATION}

The study is approved by the Regional Committee Medical Research Ethics in Mid-Norway and The ACU Human Research Ethics Committee and has its origin in the Declaration of Helsinki and is consistent with ICH/ Good Clinical Practice and applicable regulatory requirements. All protocol modification must be approved by the Regional Committee Medical Research Ethics in Mid-Norway and by The ACU Human Research Ethics Committee.
We will publish the results from the study as peerreviewed articles in international journals. Our results will also be communicated through the Norwegian society of infertility, through social media channels as well as at national and international conferences.

\section{DISCUSSION}

Infertility and risk of lifestyle-related diseases (obesity, metabolic, cardiovascular and psychological diseases) are the main health challenges associated with PCOS, both for the individuals affected by the syndrome, the healthcare systems and society. ${ }^{24}$ To date, no treatment fully reverses or cures the symptoms of PCOS ${ }^{40}$ and lifestyle interventions are recommended as first-line therapy according to recent guidelines. ${ }^{42}$ Nevertheless, there is limited research available to give women with PCOS sufficient recommendations on physical activity and exercise. Some studies have demonstrated positive effects in reproductive outcomes after exercise, with concomitant improvements in insulin sensitivity and/or visceral fat. ${ }^{20-24}$ However, more knowledge is needed about the underlying mechanisms for improvement in fertility after exercise training, as discussed in several systematic reviews on lifestyle interventions and fertility. ${ }^{1255354}$ HIT has shown to be time efficient, safe and well tolerated, with positive effects on metabolic and cardiovascular risk factors in several studies. ${ }^{2955} 56$ Larger studies investigating the adherence to HIT are lacking among women in general, and among women with PCOS in particular. This is of great interest, as we need to find exercise and lifestyle programmes that are feasible over time and that can be easily implemented in everyday life. Exercise programmes that are efficient in a research setting are not necessarily effective in a reallife setting. Thus, there is a great need for studies with a longer intervention and follow-up period.

Our trial has some limitations. The use of menstrual frequency as a proxy for ovulation/reproductive function is one of these. Due to practical reasons, we are not able to undertake ovulation monitoring. The Inbody 720 and DXA are both reliable methods to measure fat-free mass (FFM), body fat percentage (BF\%) and fat mass (FM) but it has been shown that the InBody 720 overestimates FFM and underestimates BF\% and FM compared with DXA.$^{57}$ For each individual participant, the same measurement method will be used (InBody 720 in Norway and DXA in Australia) and our purpose is to evaluate changes in body composition (ie, delta changes). Assessing enjoyment of exercise only at the supervised sessions could imply a bias. On the supervised sessions, the participants will receive motivational support from the researchers and potentially also social support from other participants that can affect their ratings of enjoyment. The enjoyment ratings are, however, implemented to compare the perceived enjoyment of the two HIT protocols. The semi-supervised nature of the trial could imply that some participants choose to undertake all sessions supervised, whereas others choose to exercise at home for most 
sessions. We chose to implement a semi-supervised exercise programme to account for the individual preferences of the participants and to be able to include women living further away from the study centres. Such protocols can both control the exercise intervention and motivate participants, and at the same time give more flexibility based on participants' preferences.

The IMPROV-IT trial will determine the effect of HIT on reproductive, cardiovascular, metabolic and psychological health in women with PCOS. The trial will contribute evidence of more specific exercise protocols in the treatment and management of PCOS for clinicians and answer some of the current limitations addressed by both the International evidence-based guidelines for the assessment and management of $\mathrm{PCOS}^{4}$ and several systematic reviews. ${ }^{2} 122558$ This trial is highly relevant and important as PCOS is a major burden for the women with PCOS, the health services and society. As for now, we do not have an optimal treatment.

\section{Trial status}

We have recruited 64 women with PCOS to the IMPROV-IT study, with the last included participant in March 2019. The last follow-up will be in March 2020.

\section{Author affiliations}

${ }^{1}$ Department of Circulation and Medical Imaging, Faculty of Medicine and Health Sciences, Norwegian University of Science and Technology, Trondheim, Norway

${ }^{2}$ Department of Obstetrics and Gynaecology, St Olavs Hospital, Trondheim University Hospital, Trondheim, Norway

${ }^{3}$ Exercise and Nutrition Research Programme, Mary Mackillop Institute for Health Research, Australian Catholic University, Melbourne, Victoria, Australia

${ }^{4}$ Research Institute for Sport and Exercise Science (RISES), Liverpool John Moores University, Liverpool, UK

${ }^{5}$ Department of Clinical and Molecular Medicine, Faculty of Medicine and Health Sciences, Norwegian University of Science and Technology, Trondheim, Norway

${ }^{6}$ Unit of Applied Clinical Research, Department of Public Health and Nursing, Faculty of Medicine and Health Sciences, Norwegian University of Science and Technology, Trondheim, Norway

\section{Twitter Trine Moholdt @trinemoholdt}

Acknowledgements The Unit for Applied Clinical Research, NTNU, provided the internet-based randomisation. The following acknowledgements apply to the Trondheim site of the trial: The equipment and lab facilities for the training sessions and exercise testing will be provided by NeXT Move, Norwegian University of Science and Technology. Blood sampling and fat biopsies will be undertaken at the Clinical Research Facility, St. Olavs Hospital. Blood and adipose tissue samples are stored in the Regional Biobank $1 \circledR$ of Central Norway, with the data solution BioByte.

Contributors IAK: drafted the manuscript. IAK, TM, EV, ØS and HJ: conceived and contributed to the design of the study. IAK, SL, TM and EP coordinated the study at the two sites and supervised the exercise training. IAK, SL, TM, MAHR and EP: performed measurements on test-days. EV: provided medical advice and support during the study. All authors provided feedback and approved the final manuscript.

Funding This work was supported by the Liaison Committee for education, research and innovation in Central Norway (Grant number: 2014/23166), the Norwegian University of Science and Technology and the Australian Catholic University. Next Move is funded by the Faculty of Medicine and Health Sciences, NTNU and Central Norway Regional Health Authority.

\section{Competing interests None declared.}

Patient consent for publication Not required.

Provenance and peer review Not commissioned; externally peer reviewed.
Open access This is an open access article distributed in accordance with the Creative Commons Attribution Non Commercial (CC BY-NC 4.0) license, which permits others to distribute, remix, adapt, build upon this work non-commercially, and license their derivative works on different terms, provided the original work is properly cited, appropriate credit is given, any changes made indicated, and the use is non-commercial. See: http://creativecommons.org/licenses/by-nc/4.0/.

ORCID iD

Ida Almenning Kiel http://orcid.org/0000-0001-9926-4994

\section{REFERENCES}

1 Yildiz BO, Bozdag G, Yapici Z, et al. Prevalence, phenotype and cardiometabolic risk of polycystic ovary syndrome under different diagnostic criteria. Hum Reprod 2012;27:3067-73.

2 Lim SS, Hutchison SK, Van Ryswyk E, et al. Lifestyle changes in women with polycystic ovary syndrome. Cochrane Database Syst Rev 2019;3:Cd007506.

3 Bozdag G, Mumusoglu S, Zengin D, et al. The prevalence and phenotypic features of polycystic ovary syndrome: a systematic review and meta-analysis. Hum Reprod 2016;31:2841-55.

4 Teede $\mathrm{H}$, Misso M, Costello M, et al. International evidence-based guideline for the assessment and management of polycystic ovary syndrome 2018. Melbourne, Australia: Monash University, 2018.

5 Goodarzi MO, Dumesic DA, Chazenbalk G, et al. Polycystic ovary syndrome: etiology, pathogenesis and diagnosis. Nat Rev Endocrinol 2011;7:219-31.

6 Joham AE, Teede HJ, Ranasinha S, et al. Prevalence of infertility and use of fertility treatment in women with polycystic ovary syndrome: data from a large community-based cohort study. J Womens Health 2015;24:299-307.

7 Giallauria F, Orio F, Palomba S, et al. Cardiovascular risk in women with polycystic ovary syndrome. J Cardiovasc Med 2008;9:987-92.

8 Teede H, Deeks A, Moran L. Polycystic ovary syndrome: a complex condition with psychological, reproductive and metabolic manifestations that impacts on health across the lifespan. BMC Med 2010;8:41.

9 Moran LJ, Pasquali R, Teede HJ, et al. Treatment of obesity in polycystic ovary syndrome: a position statement of the androgen excess and polycystic ovary syndrome Society. Fertil Steril 2009;92:1966-82.

10 Diamanti-Kandarakis E, Papavassiliou AG. Molecular mechanisms of insulin resistance in polycystic ovary syndrome. Trends $\mathrm{Mol}$ Med 2006;12:324-32.

11 Corbould A. Insulin resistance in skeletal muscle and adipose tissue in polycystic ovary syndrome: are the molecular mechanisms distinct from type 2 diabetes? Panminerva Med 2008;50:279-94.

12 Harrison CL, Lombard CB, Moran LJ, et al. Exercise therapy in polycystic ovary syndrome: a systematic review. Hum Reprod Update 2011;17:171-83.

13 Cassar S, Misso ML, Hopkins WG, et al. Insulin resistance in polycystic ovary syndrome: a systematic review and meta-analysis of euglycaemic-hyperinsulinaemic clamp studies. Hum Reprod 2016;31:2619-31.

14 Stepto NK, Cassar S, Joham AE, et al. Women with polycystic ovary syndrome have intrinsic insulin resistance on euglycaemichyperinsulaemic clamp. Hum Reprod 2013;28:777-84.

15 Villa J, Pratley RE. Adipose tissue dysfunction in polycystic ovary syndrome. Curr Diab Rep 2011;11:179-84.

16 Spritzer PM, Lecke SB, Satler F, et al. Adipose tissue dysfunction, adipokines, and low-grade chronic inflammation in polycystic ovary syndrome. Reproduction 2015;149:R219-27.

17 Thomson RL, Buckley JD, Lim SS, et al. Lifestyle management improves quality of life and depression in overweight and obese women with polycystic ovary syndrome. Fertil Steril 2010;94:1812-6.

18 Greenwood EA, Noel MW, Kao C-N, et al. Vigorous exercise is associated with superior metabolic profiles in polycystic ovary syndrome independent of total exercise expenditure. Fertil Steril 2016;105:486-93.

19 Lamb JD, Johnstone EB, Rousseau J-A, et al. Physical activity in women with polycystic ovary syndrome: prevalence, predictors, and positive health associations. Am J Obstet Gynecol 2011;204:352. e1-352.e6.

20 Vigorito C, Giallauria F, Palomba S, et al. Beneficial effects of a threemonth structured exercise training program on cardiopulmonary functional capacity in young women with polycystic ovary syndrome. $J$ Clin Endocrinol Metab 2007;92:1379-84.

21 Palomba S, Giallauria F, Falbo A, et al. Structured exercise training programme versus hypocaloric hyperproteic diet in obese polycystic 
ovary syndrome patients with anovulatory infertility: a 24-week pilot study. Hum Reprod 2008;23:642-50.

22 Turan V, Mutlu EK, Solmaz U, et al. Benefits of short-term structured exercise in non-overweight women with polycystic ovary syndrome: a prospective randomized controlled study. J Phys Ther Sci 2015;27:2293-7.

23 Nybacka Åsa, Carlström K, Ståhle A, et al. Randomized comparison of the influence of dietary management and/or physical exercise on ovarian function and metabolic parameters in overweight women with polycystic ovary syndrome. Fertil Steril 2011;96:1508-13.

24 Redman LM, Elkind-Hirsch K, Ravussin E. Aerobic exercise in women with polycystic ovary syndrome improves ovarian morphology independent of changes in body composition. Fertil Steril 2011;95:2696-9.

25 Hakimi O, Cameron L-C. Effect of exercise on ovulation: a systematic review. Sports Med 2017;47:1555-67.

26 Thomson RL, Buckley JD, Noakes M, et al. The effect of a hypocaloric diet with and without exercise training on body composition, cardiometabolic risk profile, and reproductive function in overweight and obese women with polycystic ovary syndrome. J Clin Endocrinol Metab 2008;93:3373-80.

27 Hutchison SK, Stepto NK, Harrison CL, et al. Effects of exercise on insulin resistance and body composition in overweight and obese women with and without polycystic ovary syndrome. J Clin Endocrinol Metab 2011;96:E48-56.

28 Dubé JJ, Allison KF, Rousson V, et al. Exercise dose and insulin sensitivity: relevance for diabetes prevention. Med Sci Sports Exerc 2012;44:793-9.

29 Tjønna AE, Lee SJ, Rognmo Øivind, et al. Aerobic interval training versus continuous moderate exercise as a treatment for the metabolic syndrome: a pilot study. Circulation 2008;118:346-54.

30 Cassidy S, Thoma C, Houghton D, et al. High-Intensity interval training: a review of its impact on glucose control and cardiometabolic health. Diabetologia 2017;60:7-23.

31 Weston KS, Wisløff U, Coombes JS. High-Intensity interval training in patients with lifestyle-induced cardiometabolic disease: a systematic review and meta-analysis. Br J Sports Med 2014;48:1227-34.

32 Almenning I, Rieber-Mohn A, Lundgren KM, et al. Effects of high intensity interval training and strength training on metabolic, cardiovascular and hormonal outcomes in women with polycystic ovary syndrome: a pilot study. PLoS One 2015;10:e0138793.

33 Helgerud J, Høydal K, Wang E, et al. Aerobic high-intensity intervals improve VO2max more than moderate training. Med Sci Sports Exerc 2007;39:665-71.

34 Moholdt T, Aamot IL, Granøien I, et al. Aerobic interval training increases peak oxygen uptake more than usual care exercise training in myocardial infarction patients: a randomized controlled study. Clin Rehabil 2012;26:33-44.

35 Gillen JB, Gibala MJ. Is high-intensity interval training a time-efficient exercise strategy to improve health and fitness? Appl Physiol Nutr Metab 2014;39:409-12.

36 Conte F, Banting L, Teede HJ, et al. Mental health and physical activity in women with polycystic ovary syndrome: a brief review. Sports Med 2015;45:497-504

37 Little JP, Gillen JB, Percival ME, et al. Low-Volume high-intensity interval training reduces hyperglycemia and increases muscle mitochondrial capacity in patients with type 2 diabetes. $J$ Appl Physiol 2011;111:1554-60.

38 Rotterdam ESHRE/ASRM-Sponsored PCOS consensus workshop group. Revised 2003 consensus on diagnostic criteria and longterm health risks related to polycystic ovary syndrome (PCOS). Hum Reprod 2004;19:41-7.

39 Roberts-Wilson TK, Spencer JB, Fantz CR. Using an algorithmic approach to secondary amenorrhea: avoiding diagnostic error. Clin Chim Acta 2013;423:56-61.
40 Practice Committee of American Society for Reproductive Medicine. Current evaluation of amenorrhea. Fertil Steril 2008;90:S219-25.

41 Ferriman D, Gallwey JD. Clinical assessment of body hair growth in women. J Clin Endocrinol Metab 1961;21:1440-7.

42 Berglund IJ, Sørås SE, Relling BE, et al. The relationship between maximum heart rate in a cardiorespiratory fitness test and in a maximum heart rate test. J Sci Med Sport 2019;22:607-10.

43 Matthews DR, Hosker JP, Rudenski AS, et al. Homeostasis model assessment: insulin resistance and beta-cell function from fasting plasma glucose and insulin concentrations in man. Diabetologia 1985;28:412-9.

44 Jeukendrup AE, Wallis GA. Measurement of substrate oxidation during exercise by means of gas exchange measurements. Int $J$ Sports Med 2005;26 Suppl 1:S28-37.

45 Schindelin J, Arganda-Carreras I, Frise E, et al. Fiji: an open-source platform for biological-image analysis. Nat Methods 2012;9:676-82.

46 Cronin L, Guyatt G, Griffith L, et al. Development of a healthrelated quality-of-life questionnaire (PCOSQ) for women with polycystic ovary syndrome (PCOS). J Clin Endocrinol Metab 1998;83:1976-87.

47 Thijssen DHJ, Black MA, Pyke KE, et al. Assessment of flowmediated dilation in humans: a methodological and physiological guideline. Am J Physiol Heart Circ Physiol 2011;300:H2-12.

48 Touboul P-J, Hennerici MG, Meairs S, et al. Mannheim carotid intima-media thickness and plaque consensus (2004-20062011). An update on behalf of the Advisory Board of the 3rd, 4th and 5th watching the risk symposia, at the 13th, 15th and 20th European stroke conferences, Mannheim, Germany, 2004, Brussels, Belgium, 2006, and Hamburg, Germany, 2011. Cerebrovasc Dis 2012;34:290-6.

49 Kendzierski D, DeCarlo KJ, Kendzierski D. Physical activity enjoyment scale: two validation studies. J Sport Exerc Psychol 1991;13:50-64

50 Chen MJ, Fan X, Moe ST. Criterion-related validity of the Borg ratings of perceived exertion scale in healthy individuals: a meta-analysis. $J$ Sports Sci 2002;20:873-99.

51 Hoeger KM, Kochman L, Wixom N, et al. A randomized, 48-week, placebo-controlled trial of intensive lifestyle modification and/ or metformin therapy in overweight women with polycystic ovary syndrome: a pilot study. Fertil Steril 2004;82:421-9.

52 Teede HJ, Misso ML, Costello MF, et al. Recommendations from the International evidence-based guideline for the assessment and management of polycystic ovary syndrome. Hum Reprod 2018;33:1602-18.

53 Haqq L, McFarlane J, Dieberg G, et al. Effect of lifestyle intervention on the reproductive endocrine profile in women with polycystic ovarian syndrome: a systematic review and meta-analysis. Endocr Connect 2014;3:36-46.

54 Thomson RL, Buckley JD, Brinkworth GD. Exercise for the treatment and management of overweight women with polycystic ovary syndrome: a review of the literature. Obes Rev 2011;12:e202-10.

55 Gillen JB, Little JP, Punthakee Z, et al. Acute high-intensity interval exercise reduces the postprandial glucose response and prevalence of hyperglycaemia in patients with type 2 diabetes. Diabetes Obes Metab 2012;14:575-7.

56 Earnest CP, Lupo M, Thibodaux J, et al. Interval training in men at risk for insulin resistance. Int J Sports Med 2013;34:355-63.

57 McLester CN, Nickerson BS, Kliszczewicz BM, et al. Reliability and agreement of various InBody body composition Analyzers as compared to dual-energy X-ray absorptiometry in healthy men and women. J Clin Densitom 2018. doi:10.1016/j.jocd.2018.10.008. [Epub ahead of print: 03 Nov 2018].

58 Moran LJ, Hutchison SK, Norman RJ, et al. Lifestyle changes in women with polycystic ovary syndrome. Cochrane Database Syst Rev 2011;41:CD007506. 